\title{
On Intersection Graphs of Segments with Prescribed Slopes
}

\author{
Jakub Černý, Daniel Král’^, Helena Nyklová, and Ondřej Pangrác \\ Department of Applied Mathematics and \\ Institute for Theoretical Computer Science (ITI) ${ }^{\star \star}$, \\ Charles University, Malostranské nám. 25, \\ 11800 Prague, Czech Republic, \\ \{cerny, kral, nyklova, pangrac\}@kam.ms.mff.cuni.cz
}

\begin{abstract}
We study intersection graphs of segments with prescribed slopes in the plane. A sufficient and necessary condition on tuples of slopes in order to define the same class of graphs is presented for both the possibilities that the parallel segments can or cannot overlap. Classes of intersection graphs of segments with four slopes are fully described; in particular, we find an infinite set of quadruples of slopes which define mutually distinct classes of intersection graphs of segments with those slopes.
\end{abstract}

\section{Introduction}

Intersection graphs of various types of geometric objects attract attention of researchers and find their applications in many areas of computer science. An intersection graph of a set of geometric objects is the graph whose vertices correspond to the objects of the set and two of them are joined by an edge if and only if the corresponding objects intersect. Intersection graphs of chords in a circle 25]6, of arcs of a circle [16, of segments 31913/1415], of simple curves in the plane [4810], of convex sets in the plane and others [111] have been studied intensively. We focus our attention on intersection graphs of segments with prescribed slopes in the plane (see [13]) in this paper. Such classes of graphs have been widely studied. A result of de Fraysseix et al. 7] says that each bipartite planar graph can be represented as an intersection graph of segments with two slopes such that no two segments have an interior point in common.

We study intersection graphs of segments such that the slopes of the segments of one of its realizations are among prescribed slopes $\alpha_{1}, \ldots, \alpha_{k}$ (precise definitions of the classes which interest us can be found in Section 2). We study both the classes for which parallel segments can or cannot overlap. The classes of intersection graphs of non-overlapping segments were defined and investigated by Kratochvíl and Matoušek in [13]. They proved that for $k=3$ this class

* Supported in part by KONTAKT ME337/99

** This research was supported by GAUK 158/99 and GAČR 201/99/0242. Institute for Theoretical Computer Science (ITI) is supported as project LN00A056 by the Ministry of Education of Czech Republic.

P. Mutzel, M. Jünger, and S. Leipert (Eds.): GD 2001, LNCS 2265, pp. 261-271, 2002.

(C) Springer-Verlag Berlin Heidelberg 2002 
of graphs is the same (regardless the choice of the slopes) as the class of intersection graphs of segments with three distinct slopes (the slopes are not prescribed in this case). On the other hand, they also proved in [13] that this does not hold for $k=4$. We prove a sufficient and necessary condition on the $k$-tuples of slopes $\alpha_{1}, \ldots, \alpha_{k}$ and $\beta_{1}, \ldots, \beta_{k}$ in order to define equal classes of intersection graphs of segments with their slopes among these $k$-tuples (Theorem 1 ) for both the cases when the segments can and cannot overlap. We further prove that the classes of graphs for distinct choices of slopes are either equal or, neither one of them is a subset of the other (Theorem 2), even if one of them is a class of overlapping segments and the other is a class of non-overlapping segments. Classes of intersection graphs of segments with four distinct slopes are fully described in Theorem 3 and in Corollary 1 Corollary 1 answers the question presented by Jan Kratochvíl during the 1st Graph Drawing conference in Paris in 1993: "Are there only finitely many distinct classes of intersection graphs of segments with prescribed four distinct slopes?" This question can be rephrased in terms introduced in Section 2 as follows: "Are there only finitely many mutually non-equivalent quadruples of slopes?" Corollary 1 provide us infinite number of distinct classes of intersection graphs of segments with prescribed quadruples of slopes.

We give definitions and introduce notation used in the paper together with the previously known results in Section 2. We study when two $k$-tuples of slopes define the same class of graphs in Section 3 We present an example of a graph which can be realized as an intersection graph of non-overlaping segments with a given $k$-tuple of slopes and which cannot be realized by possibly overlapping segments with slopes of another given $k$-tuple in Section 4 We combine the results of Section 3 and Section 4 in Section 5 and we state our main results.

\section{Definitions and Basic Properties}

A graph is a simple undirected graph in the whole paper; if $G$ is a graph, we write $V(G)$ for its vertex set and $E(G)$ for its edge set. Let $C$ be a class of sets indexed by vertices of $G$. $C$ is an intersection realization of a graph $G$ if $c_{u} \cap c_{v} \neq \emptyset$ if and only if $u v \in E(G)$ where $c_{u}\left(c_{v}\right)$ is the set of $C$ indexed by the vertex $u$ $(v)$. A graph is an intersection graph with respect to a certain class of objects if it can be realized by objects within this class. We study the following classes of intersection graphs of segments which were defined in [13:

- SEG is the class of intersection graphs of segments in the plane, i.e. those graphs for which there exists a set of segments $C$ such that $C$ is an intersection realization of $G$ (parallel segments may overlap).

- PURE-SEG is the class of intersection graphs of non-overlapping segments, i.e. those graphs for which there exists a set of segments $C$ such that the parallel segments of $C$ are disjoint (i.e. do not overlap) and $C$ is an intersection realization of $G$.

$-k-\operatorname{DIR}\left(\alpha_{1}, \ldots, \alpha_{k}\right)$ is the class of intersection graphs of segments with slopes $\alpha_{1}, \ldots, \alpha_{k}$ in the plane, i.e. those graphs for which there exists a set of segments $C$ with slopes among $\alpha_{1}, \ldots, \alpha_{k}$ such that $C$ is an intersection real- 
ization of $G$ (parallel segments may overlap). We assume w.l.o.g. throughout the paper that $0=\alpha_{1}<\ldots<\alpha_{k}<\pi$.

$-k$ - PURE-DIR $\left(\alpha_{1}, \ldots, \alpha_{k}\right)$ is the class of intersection graphs of non-overlapping segments with slopes $\alpha_{1}, \ldots, \alpha_{k}$ in the plane i.e. those graphs for which there exists a set of segments $C$ with slopes among $\alpha_{1}, \ldots, \alpha_{k}$ such that the parallel segments of $C$ are disjoint (i.e. do not overlap) and $C$ is an intersection realization of $G$. We also assume w.l.o.g. that $0=\alpha_{1}<\ldots<$ $\alpha_{k}<\pi$ in the paper.

$-k-\mathrm{DIR}=\cup_{\alpha_{1}, \ldots, \alpha_{k}} k-\operatorname{DIR}\left(\alpha_{1}, \ldots, \alpha_{k}\right)$ is the class of intersection graphs of segments of at most $k$ slopes.

$-k-\mathrm{PURE}-\mathrm{DIR}=\cup_{\alpha_{1}, \ldots, \alpha_{k}} k-\operatorname{DIR}\left(\alpha_{1}, \ldots, \alpha_{k}\right)$ is the class of intersection graphs of non-overlapping segments of at most $k$ slopes.

Note that SEG $=\cup_{k=1}^{\infty} k-\mathrm{DIR}$ and PURE-SEG $=\cup_{k=1}^{\infty} k-\mathrm{PURE-DIR}$. It is also clear that $k-\operatorname{PURE-DIR}\left(\alpha_{1}, \ldots, \alpha_{k}\right) \subset k-\operatorname{DIR}\left(\alpha_{1}, \ldots, \alpha_{k}\right)$ for an arbitrary choice of $\alpha_{1}, \ldots, \alpha_{k}$ and $k-$ PURE-DIR $\subset k-$ DIR (both inclusions are strict).

We use linear transformations of the plane in several proofs in this paper. We describe the transformations by $2 \times 2-$ matrices of real numbers. If $A$ is such a matrix and if $[x, y]$ is a point in the plane with the coordinates equal to $x$ and $y$, then the transformation described by $A$ maps the point $[x, y]$ to the point $A[x, y]$ with its coordinates equal to $a_{11} x+a_{12} y$ and $a_{21} x+a_{22} y$.

We say that two $k$-tuples of slopes $\alpha_{1}, \ldots, \alpha_{k}$ and $\beta_{1}, \ldots, \beta_{k}$ are equivalent if and only if $k-\operatorname{PURE-DIR}\left(\alpha_{1}, \ldots, \alpha_{k}\right)=k-\operatorname{PURE}-\operatorname{DIR}\left(\beta_{1}, \ldots, \beta_{k}\right)$. Later (consult Theorem 11), we show that the latter condition is equivalent to $k-\operatorname{DIR}\left(\alpha_{1}, \ldots, \alpha_{k}\right)=k-\operatorname{DIR}\left(\beta_{1}, \ldots, \beta_{k}\right)$. We say that the $k$-tuple of slopes $\alpha_{1}, \ldots, \alpha_{k}$ can be transformed to the $k$-tuple of slopes $\beta_{1}, \ldots, \beta_{k}$ if there exists an affine transformation of the plane which transforms one of them to the other, i.e. the following holds: There exists a regular square matrix $A$ of size two such that $\left\{\left[t \cos \alpha_{i}, t \sin \alpha_{i}\right], t \in \mathrm{R}, 1 \leq i \leq k\right\}=\left\{A\left[t \cos \beta_{i}, t \sin \beta_{i}\right], t \in \mathrm{R}, 1 \leq i \leq k\right\}$. Note that if $\alpha_{1}, \ldots, \alpha_{k}$ can be transformed to $\beta_{1}, \ldots, \beta_{k}$, then $\beta_{1}, \ldots, \beta_{k}$ can be transformed to $\alpha_{1}, \ldots, \alpha_{k}$ (consider the inverse matrix).

The following relations between distinct classes of intersection graphs of nonoverlapping segments were proved by Kratochvíl and Matoušek in [13]:

$-k-\mathrm{PURE}-\mathrm{DIR} \subset(k+1)-\mathrm{PURE}-\mathrm{DIR}$ for all $k \geq 1$ (the inclusion is strict).

$-2-\mathrm{PURE}-\mathrm{DIR}=2-\operatorname{PURE}-\mathrm{DIR}\left(\alpha_{1}, \alpha_{2}\right)$ for any $0 \leq \alpha_{1}<\alpha_{2}<\pi$.

-3 -PURE-DIR $=3-\operatorname{PURE-DIR}\left(\alpha_{1}, \alpha_{2}, \alpha_{3}\right)$ for any $0 \leq \alpha_{1}<\alpha_{2}<\alpha_{3}<\pi$.

$-4-\mathrm{PURE}-\mathrm{DIR} \neq 4-\mathrm{PURE}-\mathrm{DIR}(0, \pi / 4, \pi / 2,3 \pi / 4)$.

The second and the third result can be restated in our terminology as follows: Any two pairs (triples) of slopes are equivalent.

A finite collection of lines in the plane is called a line arrangement; we suppose that no three lines of an arrangement share a common point in the whole paper. Note that two non-homeomorphic line arrangements can be intersection realizations of the same graph. The following lemma (Order Forcing Lemma), originnaly proved in [13], turned to be a really powerful tool: 
Lemma 1 (Order Forcing Lemma 12:13]). Let $L$ be an arrangement of $n$ lines in the plane and let $G$ be the intersection graph of the lines of $L$. Then there exists a graph $G^{\prime}$ with the following properties:

- $G$ is an induced subgraph of $G^{\prime}$,

- $G^{\prime} \in \mathrm{SEG}$,

- if $G \in k-\operatorname{PURE-DIR}\left(\alpha_{1}, \ldots, \alpha_{k}\right)$, then $G^{\prime} \in k-\operatorname{PURE-DIR}\left(\alpha_{1}, \ldots, \alpha_{k}\right)$ (for $k \geq 2$ ),

- for each realization of $G^{\prime}$ by segments in the plane (with possible overlapping of parallel segments), there exists a region $\Omega$ in the realization of $G^{\prime}$ and a homeomorphism $\varphi$ from the plane with the arrangement $L$ to $\Omega$ such that $\varphi\left(l_{u}\right)=r_{u}$ where $l_{u}$ is the line of $L$ corresponding to the vertex $u$ of $G$ and $r_{u}$ is the segment corresponding to the vertex $u$ of $G^{\prime}$.

We have presented this lemma here in a different (but of course equivalent) form to the one presented in 13 . This form seems to be more useful for our purposes. This lemma assures that there exists a graph $G^{\prime}$ for each line arrangement $L$, such that each realization of $G^{\prime}$ by segments in the plane contains a homeomorphic copy of the line arrangement $L$. Note that even if the realization of $G^{\prime}$ may contain overlapping parallel segments, the homeomorphic copy of the line arrangement $L$ consists of non-overlapping segments.

\section{Equivalence Results}

First, we state an easy lemma stating a sufficient condition for $k$-tuples of slopes to be equivalent:

Lemma 2. If two $k$-tuples of slopes $\alpha_{1}, \ldots, \alpha_{k}$ and $\beta_{1}, \ldots, \beta_{k}$ can be transformed one to the other, then they are equivalent.

Proof. Let $G \in k-\operatorname{PURE-DIR}\left(\alpha_{1}, \ldots, \alpha_{k}\right)$ and let $C_{\alpha}$ be its realization by segments with slopes among $\alpha_{1}, \ldots, \alpha_{k}$. Let $A$ be the matrix described in the definition of transformation between $k$-tuples of slopes. Let $C_{\beta}$ be the set containing the following segments: If $p$ is a segment of $C_{\alpha}$, then the segment $A p=\{A[x, y],[x, y] \in p\}$ belongs to the set $C_{\beta}$. Note that $A p$ and $A q$ intersect (overlap) if and only if $p$ and $q$ intersect (overlap) because $A$ is a regular matrix. Due to the choice of $A$, the slopes of segments of $C_{\beta}$ are among $\beta_{1}, \ldots, \beta_{k}$ and thus $G \in k-\operatorname{PURE}-\operatorname{DIR}\left(\beta_{1}, \ldots, \beta_{k}\right)$. This implies that $k$-PURE-DIR $\left(\alpha_{1}, \ldots, \alpha_{k}\right) \subseteq k-\operatorname{PURE-DIR}\left(\beta_{1}, \ldots, \beta_{k}\right)$. The opposite inclusion can be proved in the same way.

The following lemma provides us some canonical choice of slopes:

Lemma 3. Let $\alpha_{1}, \ldots, \alpha_{k}$ be a $k$-tuple of slopes (where $k \geq 3$ ). There exists $\pi / 2<\beta_{4}<\ldots<\beta_{k}<\pi$ such that $k-\operatorname{PURE-DIR}\left(\alpha_{1}, \ldots, \alpha_{k}\right)=$ $k-\operatorname{PURE}-\operatorname{DIR}\left(0, \pi / 4, \pi / 2, \beta_{4}, \ldots, \beta_{k}\right)$. In particular if $k$ is eqaul to 3 , then $3-\operatorname{PURE-DIR}\left(\alpha_{1}, \alpha_{2}, \alpha_{3}\right)=3-\operatorname{PURE-DIR}(0, \pi / 4, \pi / 2)$ for all $\alpha_{1}, \alpha_{2}, \alpha_{3}$. 
Proof. We can assume w.l.o.g. that $0=\alpha_{1}<\ldots<\alpha_{k}<\pi$ as stated in Section 2 Consider the following matrix $A$ :

$$
A=\left(\begin{array}{cc}
1 & -\operatorname{cotg} \alpha_{3} \\
0 & \frac{\sin \left(\alpha_{3}-\alpha_{2}\right)}{\sin \alpha_{2} \sin \alpha_{3}}
\end{array}\right)
$$

The matrix $A$ is regular, since its determinant is non-zero. Note that the following holds:

$$
\begin{gathered}
A\left[\cos \alpha_{1}, \sin \alpha_{1}\right]=A[1,0]=[1,0] \\
A\left[\cos \alpha_{2}, \sin \alpha_{2}\right]=\left[\frac{\sin \left(\alpha_{3}-\alpha_{2}\right)}{\sin \alpha_{3}}, \frac{\sin \left(\alpha_{3}-\alpha_{2}\right)}{\sin \alpha_{3}}\right] \\
A\left[\cos \alpha_{3}, \sin \alpha_{3}\right]=\left[0, \frac{\sin \left(\alpha_{3}-\alpha_{2}\right)}{\sin \alpha_{2}}\right]
\end{gathered}
$$

Thus the matrix $A$ transforms the slope $\alpha_{1}\left(\alpha_{2}, \alpha_{3}\right.$ respectively) to $0(\pi / 4$, $\pi / 2$ respectively). Let $\beta_{i}$ (where $4 \leq i \leq k$ ) be the slope which the slope $\alpha_{i}$ is transformed to. It is enough to apply Lemma 2 to the $k$-tuples $\alpha_{1}, \ldots, \alpha_{k}$ and $0, \pi / 4, \pi / 2, \beta_{4}, \ldots, \beta_{k}$ to prove the statement of the lemma. The matrices $A$ and $A^{-1}$ witness that these two $k$-tuples of slopes can be transformed one to the other.

We haven't used in the proofs of Lemma 2 and Lemma 3 that the parallel segments do not overlap; thus the corresponding lemmas for the classes of overlapping segments also hold:

Lemma 4. If two $k$-tuples of slopes $\alpha_{1}, \ldots, \alpha_{k}$ and $\beta_{1}, \ldots, \beta_{k}$ can be transformed one to the other, then $k-\operatorname{DIR}\left(\alpha_{1}, \ldots, \alpha_{k}\right)=k-\operatorname{DIR}\left(\beta_{1}, \ldots, \beta_{k}\right)$.

Lemma 5. Let $\alpha_{1}, \ldots, \alpha_{k}$ be any given $k$-tuple of slopes (for $k \geq 3$ ). Then there exists $\pi / 2<\beta_{4}<\ldots<\beta_{k}<\pi$ such that $k-\operatorname{DIR}\left(\alpha_{1}, \ldots, \alpha_{k}\right)=k-$ $\operatorname{DIR}\left(0, \pi / 4, \pi / 2, \beta_{4}, \ldots, \beta_{k}\right)$. In particular if $k=3$, then $3-\operatorname{DIR}\left(\alpha_{1}, \alpha_{2}, \alpha_{3}\right)=$ $3-\operatorname{DIR}(0, \pi / 4, \pi / 2)$ for all $\alpha_{1}, \alpha_{2}, \alpha_{3}$.

\section{Non-equivalence Results}

Lemma 6. Let $\pi / 2<\alpha_{4}<\ldots<\alpha_{k}<\pi$ and $\epsilon>0$ be given. Then, there exists a graph $G$ with three distinguished vertices $U, V$ and $W$ with the following properties:

$-G \in k-\operatorname{PURE}-\operatorname{DIR}\left(0, \pi / 4, \pi / 2, \alpha_{4}, \ldots, \alpha_{k}\right)$,

$-G \notin(k-1)-\mathrm{DIR}$,

- the segments corresponding to $U, V$ and $W$ have different slopes in any realization of $G$ by (not neceserally non-overlapping) segments with $k$ slopes 
- let $C$ be any realization of $G$ by (possibly overlapping) segments with $k$ slopes such that the segment corresponding to $U(V, W$, respectively) has the slope 0 ( $\pi / 4, \pi / 2$, respectively). Let $0<\beta_{4}<\ldots<\beta_{k}<\pi$ be the remaining slopes of the segments of $C$. It has to hold that $\pi / 2<\beta_{4}$ and $\left|\beta_{i}-\alpha_{i}\right|<\epsilon$ for $4 \leq i \leq k$.

Proof. We assume w.l.o.g. that $\epsilon$ is small enough in order that $\alpha_{4}-\epsilon>\pi / 2$ and $\alpha_{k}+\epsilon<\pi$.

We first construct line arrangements $L_{4}, \ldots, L_{k}$ and $L_{4}^{\prime}, \ldots, L_{k}^{\prime}$ and we use later them to construct the desired $G$. Let $i, 4 \leq i \leq k$, be fixed in the rest of this paragraph. Let us choose integers $p$ and $q$ such that $\operatorname{tg}\left(\alpha_{i}-\epsilon-\pi / 2\right)<p / q<$ $\operatorname{tg}\left(\alpha_{i}-\pi / 2\right)$ and let us choose integers $p^{\prime}$ and $q^{\prime}$ such that $\operatorname{tg}\left(\alpha_{i}-\pi / 2\right)<$ $p^{\prime} / q^{\prime}<\operatorname{tg}\left(\alpha_{i}+\epsilon-\pi / 2\right)$. Let $L_{i}$ and $L_{i}^{\prime}$ be the line arrangements drawn in Figure 1 the slope of the bold line is $\alpha_{i}$. Let us suppose that we have a line arrangement homeomorphic to $L_{i}$. If the slopes of horizontal (diagonal, vertical respectively) lines are precisely $0(\pi / 4, \pi / 2$ respectively), then we claim that the slope of the bold line is at least $\pi / 2+\operatorname{arctg}(p / q)$ : Let $x_{s}$ be the widths of the cells $(1 \leq s \leq p)$ and let $y_{t}$ be the heights of the cells $(1 \leq t \leq q)$ of any realization of the line arrangement $L_{i}$. The positions of diagonal lines in the cells assure that $x_{s}>y_{t}$ for all $s$ and $t$. These inequalities imply that $\frac{\sum_{s} x_{s}}{\sum_{t} y_{t}}>\frac{p}{q}$ and thus the slope of the bold line has to be at least $\pi / 2+\operatorname{arctg}(p / q)$. Similarly, the slope of the bold line in a line arrangement homeomorphic to $L_{i}^{\prime}$ has to be at most $\pi / 2+\operatorname{arctg} p^{\prime} / q^{\prime}$.

We construct a line arrangement $L$ by placing all the arrangements $L_{i}$ and $L_{i}^{\prime}$ for $4 \leq i \leq k$ to the plane (see Figure 2); we preserve the horizontal, diagonal and vertical slopes of the lines of all the arrangements and we place the arrangements with the whole lines, i.e. the non-parallel lines of distinct arrangements intersect each other. We do not care at all how the lines of distinct arrangements intersect. The lines of $L$ intersect if and only if they are not parallel. The line arrangement $L$ can be clearly realized by lines with slopes $0, \pi / 4, \pi / 2, \alpha_{4}, \ldots, \alpha_{k}$. Since $L$ contains $k$ lines with distinct slopes such that any pair of them intersects, no arrangement of lines homeomorphic to $L$ can be realized by lines with fewer than $k$ slopes; moreover, the lines in any realization with exactly $k$ slopes which is homeomorphic to $L$ are parallel if and only if they are parallel in $L$. We choose one of the vertices of the intersection graph corresponding to a horizontal line to be $U$, one of those corresponding to diagonal lines to be $V$ and one of those corresponding to vertical lines to be $W$. If the line corresponding to $U$ in a realization is horizontal, i.e. its slope is 0 , then the slopes of all the horizontal lines are 0 , since all the parallel lines must have the same slope in any realization. The same holds for diagonal and vertical lines. Thus if a homeomorphic copy of $L$ consists of lines of at most $k$ slopes and the lines corresponding to $U(V$, $W$ respectively) are horizontal (diagonal, vertical respectively), then the slopes of the lines have to differ from $0, \pi / 4, \pi / 2, \alpha_{4}, \ldots, \alpha_{k}$ by at most $\epsilon$; the bold lines of $L_{i}$ and $L_{i}^{\prime}$ have to be parallel as mentioned above in this paragraph. 

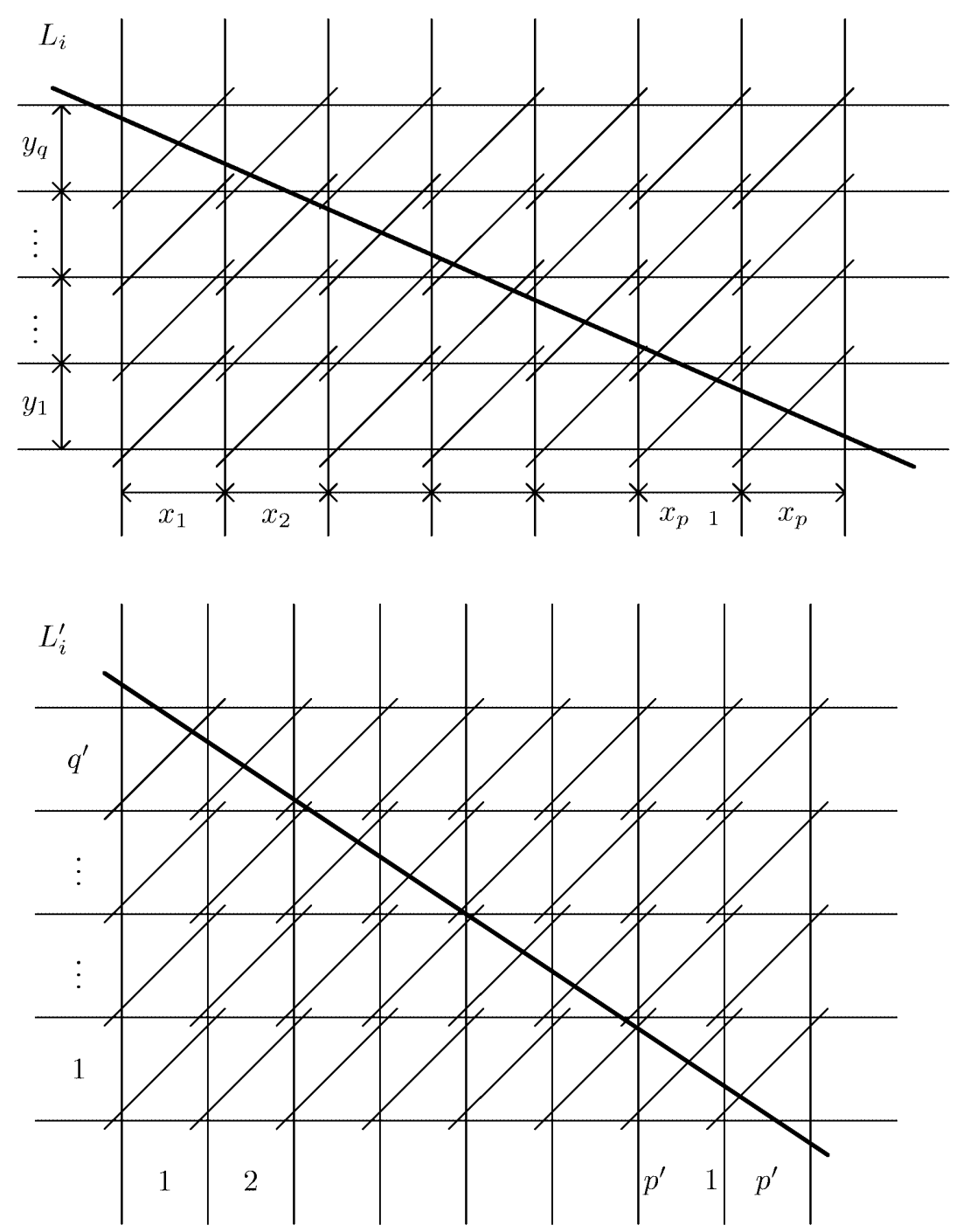

Fig. 1. The line arrangements $L_{i}$ and $L_{i}^{\prime}$. The slope of the bold line is $\alpha_{i}$.

Let $G$ be the graph for the line arrangement $L$ given by Lemma 11. The graph $G$ belongs to $k-\operatorname{PURE-DIR}\left(0, \pi / 4, \pi / 2, \alpha_{4}, \ldots, \alpha_{k}\right)$. Any realization of $G$ by lines of at most $k$ slopes (with or without possible overlapping of parallel segments) contains a homeomorphic copy of the line arrangement $L$ including the distinguished lines/vertices $U, V$ and $W$.. Thus the graph $G$ is the desired graph from the statement of the lemma. 


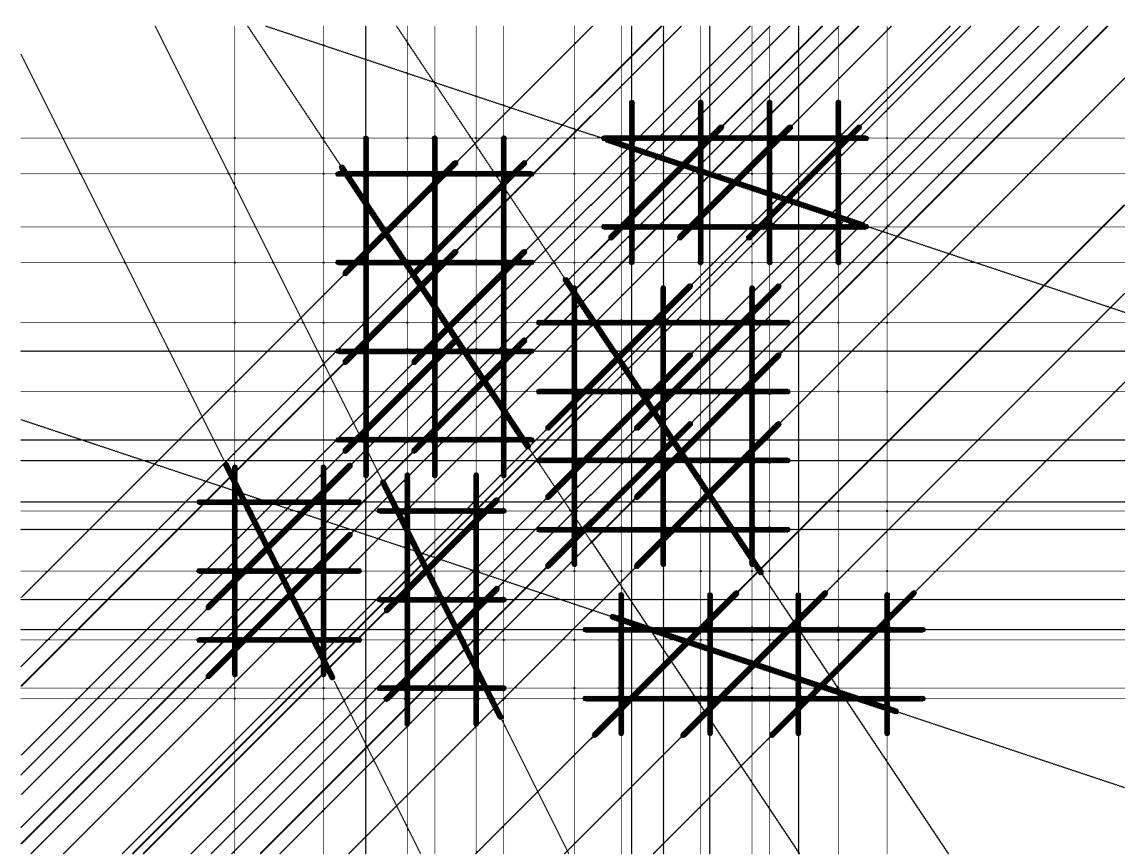

Fig. 2. Placing the line arrangements $L_{1}, \ldots, L_{k}$ and $L_{1}^{\prime}, \ldots, L_{k}^{\prime}$ to the plane. The original arrangements are marked by bold lines.

Lemma 7. Let $\alpha_{1}, \ldots, \alpha_{k}$ and $\beta_{1}, \ldots, \beta_{k}$ be any two $k$-tuples of slopes which cannot be transformed one to the other. Then, there exists a graph $G$ such that $G \in k-\operatorname{PURE}-\operatorname{DIR}\left(\alpha_{1}, \ldots, \alpha_{k}\right) \backslash k-\operatorname{DIR}\left(\beta_{1}, \ldots, \beta_{k}\right)$.

Proof. Let us assume w.l.o.g. that $\alpha_{1}=0, \alpha_{2}=\pi / 4$ and $\alpha_{3}=\pi / 2$ due to Lemma 3 Choose $\epsilon>0$ small enough in order that there exists $i$ such that $\left|\beta_{i}^{\prime}-\alpha_{i}\right|>\epsilon$ for any $k$-tuple $0, \pi / 4, \pi / 2, \beta_{4}^{\prime}, \ldots, \beta_{k}^{\prime}$ to which the $k$-tuple $\beta_{1}, \ldots, \beta_{k}$ can be transformed. Note that there are at most $2 k$ such $k$-tuples (and thus such $\epsilon$ exists): There are $k$ ways of choosing a slope which we want to be horizontal and if the horizontal slope is chosen, there two ways of choosing a slope which we want to be the " $\pi / 4$ " one (only the slopes which neighbour with the "horizontal" one can be chosen).

Let us consider the graph $G$ from Lemma [6 for $\alpha_{4}, \ldots, \alpha_{k}$ and $\epsilon$ and let $U, V$ and $W$ be its distinguished vertices. Let us suppose that $G$ has an intersection realization by (possibly overlapping) segments with the slopes $\beta_{1}, \ldots, \beta_{k}$. Let $\beta_{i_{U}}$ $\left(\beta_{i_{V}}, \beta i_{W}\right.$ respectively) be the slope of the segment corresponding to the vertex $U$ ( $V, W$ respectively). The indices $i_{U}, i_{V}$ and $i_{W}$ of the slopes are mutually distinct due to Lemma [6] Apply the transformation of the plane transforming $\beta_{i_{U}}$ to 0 , $\beta_{i_{V}}$ to $\pi / 4$ and $\beta_{i_{W}}$ to $\pi / 2$ - see the proof of Lemma 2 for details of the construction of this transformation. Let $0, \pi / 4, \pi / 2, \beta_{4}^{\prime}, \ldots, \beta_{k}^{\prime}$ be the slopes $\beta_{1}, \ldots, \beta_{k}$ 
after performing this transformation. But the properties of any realization of $G$ by segments which are stated in Lemma 6 contradict our choice of $\epsilon$ unless $\alpha_{1}, \ldots, \alpha_{k}$ can be transformed to $\beta_{1}, \ldots, \beta_{k}$. Thus such realization of $G$ by segments cannot exist and $G \in k-\operatorname{PURE}-\operatorname{DIR}\left(\alpha_{1}, \ldots, \alpha_{k}\right) \backslash k-\operatorname{DIR}\left(\beta_{1}, \ldots, \beta_{k}\right)$.

\section{$5 \quad$ Main Results}

We present our main results in this section. First, we state a necessary and sufficient condition for two $k$-tuples to be equivalent:

Theorem 1. The following statements for two $k$-tuples of slopes $\alpha_{1}, \ldots, \alpha_{k}$ and $\beta_{1}, \ldots, \beta_{k}$ are equivalent:

- The $k$-tuples $\alpha_{1}, \ldots, \alpha_{k}$ and $\beta_{1}, \ldots, \beta_{k}$ can be transfomred one to the other.

- The $k$-tuples $\alpha_{1}, \ldots, \alpha_{k}$ and $\beta_{1}, \ldots, \beta_{k}$ are equivalent, i.e. the following holds: $k-\operatorname{PURE-DIR}\left(\alpha_{1}, \ldots, \alpha_{k}\right)=k-\operatorname{PURE-DIR}\left(\beta_{1}, \ldots, \beta_{k}\right)$.

$-k-\operatorname{DIR}\left(\alpha_{1}, \ldots, \alpha_{k}\right)=k-\operatorname{DIR}\left(\beta_{1}, \ldots, \beta_{k}\right)$.

Proof. If they can be transformed one to the other, they are equivalent due to Lemma 2, the corresponding claim for overlapping version of the classes is due to Lemma 4. If they cannot be transformed, they are non-equivalent for both the versions of the classes due to Lemma 7 and due to simple fact that $k-\operatorname{PURE-DIR}\left(\alpha_{1}, \ldots, \alpha_{k}\right) \subset k-\operatorname{DIR}\left(\alpha_{1}, \ldots, \alpha_{k}\right)$.

We describe the relation between classes of intersection graphs of segments of prescribed slopes in the next theorem:

Theorem 2. Let $\alpha_{1}, \ldots, \alpha_{k}$ and $\beta_{1}, \ldots, \beta_{k}$ be two $k$-tuples. Then the following holds:

- If the $k$-tuples of slopes $\alpha_{1}, \ldots, \alpha_{k}$ and $\beta_{1}, \ldots, \beta_{k}$ can be transformed one to the other, then $k-\operatorname{PURE-DIR}\left(\alpha_{1}, \ldots, \alpha_{k}\right)=k-\operatorname{PURE-DIR}\left(\beta_{1}, \ldots, \beta_{k}\right)$ and $k-\operatorname{DIR}\left(\alpha_{1}, \ldots, \alpha_{k}\right)=k-\operatorname{DIR}\left(\beta_{1}, \ldots, \beta_{k}\right)$.

- If the $k$-tuples of slopes $\alpha_{1}, \ldots, \alpha_{k}$ and $\beta_{1}, \ldots, \beta_{k}$ cannot be transformed one to the other, then $k-\operatorname{PURE-DIR}\left(\alpha_{1}, \ldots, \alpha_{k}\right) \subset k-\operatorname{DIR}\left(\alpha_{1}, \ldots, \alpha_{k}\right) \nsubseteq k-$ $\operatorname{DIR}\left(\beta_{1}, \ldots, \beta_{k}\right), k-\operatorname{PURE-DIR}\left(\alpha_{1}, \ldots, \alpha_{k}\right) \nsubseteq \notin k-\operatorname{PURE}-\operatorname{DIR}\left(\beta_{1}, \ldots, \beta_{k}\right)$.

Proof. If the $k$-tuples of slopes can be transformed one to the other, they are equivalent and thus the corresponding classes of intersection graph of segments are equal as stated in Theorem 1. If they cannot be transformed one to the other, Lemma 7 provides a graph which witness the second statement.

Theorem 3. Let $0=\alpha_{1}<\alpha_{2}<\alpha_{3}<\alpha_{4}<\pi$ be any quadruple of slopes. There exists exactly one $\beta, \pi / 2<\beta \leq 3 \pi / 4$ such that the quadruples $\alpha_{1}, \alpha_{2}, \alpha_{3}, \alpha_{4}$ and $0, \pi / 4, \pi / 2, \beta$ are equivalent. 
Proof. Lemma 3 assures existence of $\pi / 2<\beta^{\prime}<\pi$ such that the quadruples $\alpha_{1}, \alpha_{2}, \alpha_{3}, \alpha_{4}$ and $0, \pi / 4, \pi / 2, \beta^{\prime}$ are equivalent. In case that $\beta^{\prime}>3 \pi / 4$, consider the following matrix:

$$
A=\left(\begin{array}{cc}
-1 & 0 \\
0 & -\operatorname{cotg} \beta^{\prime}
\end{array}\right)
$$

The transformation described by $A$ transforms the quadruple $0, \pi / 4, \pi / 2, \beta^{\prime}$ to $0, \pi / 4, \pi / 2,3 \pi / 2-\beta^{\prime}$. Thus choosing $\beta=3 \pi / 2-\beta^{\prime}$ assures the existence of $\pi / 2<$ $\beta \leq 3 \pi / 4$.

It remains to prove that such $\beta$ is unique. There are exactly eight matrices (up to multiplication by a constant) which transform the quadruple $0, \pi / 4, \pi / 2, \beta$ to a quadruple $0, \pi / 4, \pi / 2, \gamma$ where $\pi / 2<\gamma<\pi$ :

$$
\begin{aligned}
& \left(\begin{array}{ll}
1 & 0 \\
0 & 1
\end{array}\right) \quad\left(\begin{array}{cr}
1 & -\operatorname{cotg} \beta \\
\operatorname{cotg} \beta & -\operatorname{cotg} \beta
\end{array}\right) \quad\left(\begin{array}{cc}
0 & 1-\operatorname{cotg} \beta \\
\operatorname{tg} \beta-1 & 0
\end{array}\right)\left(\begin{array}{lc}
-1 & 1 \\
-1 & \operatorname{cotg} \beta
\end{array}\right) \\
& \left(\begin{array}{ll}
0 & 1 \\
1 & 0
\end{array}\right) \quad\left(\begin{array}{cr}
\operatorname{cotg} \beta-\operatorname{cotg} \beta \\
1 & -\operatorname{cotg} \beta
\end{array}\right) \quad\left(\begin{array}{cc}
\operatorname{tg} \beta-1 & 0 \\
0 & 1-\operatorname{cotg} \beta
\end{array}\right) \quad\left(\begin{array}{cc}
-1 \operatorname{cotg} \beta \\
-1 & 1
\end{array}\right)
\end{aligned}
$$

It is a matter of routine calculation to check that the only quadruples which can be obtained through these transformations are actually only $0, \pi / 4, \pi / 2, \beta$ and $0, \pi / 4, \pi / 2,3 \pi / 2-\beta$. This proves the unicity of $\beta$.

The immediate previously promised corollary is the following:

Corollary 1. The classes of graphs 4 - PURE-DIR $(0, \pi / 4, \pi / 2, \alpha)$ for $\pi / 2<$ $\alpha \leq 3 \pi / 4$ are mutually distinct and any class $4-\operatorname{PURE-DIR}\left(\beta_{1}, \beta_{2}, \beta_{3}, \beta_{4}\right)$ is equal to exactly one of them.

Note that statements of Corollary 1 can be also formulated for 4-DIR class of graphs (the one with overlapping parallel segments) in the same way.

Acknowledgement. Attention of the authors to intersection graphs of geometric objects were attracted by Jan Kratochvíl who has given lectures on the topic of intersection graphs. The authors are indebted to Jan Kratochvíl for fruitful discussion on the topic, especially on the Order forcing lemma, during his lectures.

\section{References}

1. T. Asano: Difficulty of the maximum independent set problem on intersection graphs of geometric objects, Graph theory, combinatorics and applications, vol. 1, Wiley-Intersci. Publ., 1991, pp. 9-18.

2. A. Bouchet: Reducing prime graphs and recognizing circle graphs, Combinatorica 7, 1987, pp. 243-254.

3. N. de Castro, F. J. Cobos, J. C. Dana, A. Marquez, M. Noy: Triangle-free planar graphs as segments intersection graphs, J. Kratochvil (ed.), Graph drawing, 7th international symposium, Štiřín Castle, Czech Republic, proceedings, Springer LNCS 1731, 1999, pp. 341-350. 
4. G. Ehrlich, S. Even, R. E. Tarjan: Intersection graphs of curves in the plane, J. Combinatorial Theory Ser. B 21, 1976, no. 1, 8-20.

5. J. C. Fournier: Une caracterization des graphes de cordes, C.R. Acad. Sci. Paris 286A, 1978, pp. 811-813.

6. H. de Fraysseix: A characterization of circle graphs, European Journal of Combinatorics 5, 1984, pp. 223-238.

7. H. de Fraysseix, P. Ossona de Mendez, J. Pach: Representation of planar graphs by segments, Intuitive Geometry 63, 1991, pp. 109-117.

8. M. Goljan, J. Kratochvíl, P. Kučera: String graphs, Academia, Prague 1986.

9. I. B.-A. Hartman, I. Newman, R. Ziv: On grid intersection graphs, Discrete Math. 87, 1991, no. 1 , pp. 41-52.

10. V. B. Kalinin: On intersection graphs, Algorithmic constructions and their efficiency (in Russian), Yaroslav. Gos. Univ., 1983, pp. 72-76.

11. S. Klavžar, M. Petkovšek: Intersection graphs of halflines and halfplanes, Discrete Math. 66, 1987, no. 1-2, pp. 133-137.

12. J. Kratochvíl: personal comunication.

13. J. Kratochvíl, J. Matoušek: Intersection Graphs of Segments, Journal of Combinatorial Theory, Series B, Vol. 62, No. 2, 1994, pp. 289-315.

14. J. Kratochvíl, J. Matoušek: NP-hardness results for intersection graphs, Comment. Math. Univ. Carolin. 30, 1989, pp. 761-773.

15. J. Kratochvíl, J. Nešetřil: Independent set and clique problems in intersection defined classes of graphs, Comment. Math. Univ.Carolin. 31, 1990, pp. 85-93.

16. A. C. Tucker: An algorithm for circular-arc graphs, SIAM J. Computing 31.2, 1980, pp. 211-216. 